\title{
Cinnamon Oil and Chitosan Coating on Orthopaedic Implant Surface for Prevention of Staphylococcus Epidermidis Biofilm Formation
}

\author{
R Magetsari, PhD, P Dewo, PhD, BK Saputro, MD, Z Lanodiyu, MD \\ Department of Orthopaedic and Traumatology Dr Sardjito General Hospital/Faculty of Medicine Universitas \\ Gadjah Mada, Yogyakarta, Indonesia
}

\begin{abstract}
$\mathrm{S}$. Epidermidis is among the most frequently isolated microorganisms found in -infection related to implanted devices and the formation of biofilm will be more resistant compared to the planktonic form. This study was carried out to determine the effect of coating on stainless steel orthopaedic implants surfaces with cinnamon oil and chitosan as bioadhesive to prevent biofilms formation of S. Epidermidis.The rod shaped stainless steel 316 L orthopaedic implant with $5 \mathrm{~mm}$ diameters was coated 2 times using a mixture of cinnamon oil and chitosan $3 \%$ and $2 \%$ respectively with serial concentration of cinnamon from $0.125 \%$ to $2 \%$. The coated implants were then put into tubes that contained bacterial suspension and incubated. Subsequently, the implants were washed with PBS solution followed by MTT soulution and isopropanol acid solution. that related to biofilm formation. The results were expressed in numbers which represents the absorbance level at ELISA readings on $575 \mathrm{~nm}$ (A575) wavelength.The stainless steel implant coated with chitosan and cinnamon oil $2 \%$ and $1 \%$ has lower absorbance level compared with the absorbance level of S.Epidermidis biofilm only.
\end{abstract}

This study showed that mixture of cinnamon oil and chitosan coated on the surface of stainless steel orthopaedic implant has an effect against S.Epidermidis biofilm formation with the minimum cinnamon oil concentration of $1 \%$.

Key Words:

Cinnamon oil; Staphylococcus Epidermidis; Biofilm, Chitosan; Orthopaedic Implant

\section{INTRODUCTION}

Staphylococcus Epidermidis (S. Epidermidis) as one of Coagulase-Negative Staphylococci (CoNS) is among the most frequently isolated microorganisms found related to complications following surgical implantation of prosthetic devices $^{1}$. National Nosocomial Infection Surveillance
System reported that CoNS are among the five most commonly reported pathogens in hospitals. Epidemiologically, it is also associated with prosthetic joints and other implanted devices in the community ${ }^{2}$. The incidence of infection following hip surgery using prosthetic device was around $0.06 \%-11.8 \%$ and following open fracture which underwent ORIF, the incidence of infection was around $13.9 \%{ }^{3,4}$. Bacteria that attach to implanted devices secrete extracellular polymeric substances, forming the matrix of microbial biofilms. It is a thin layer where the colonisation of bacteria will be 10-1000 fold more resistant to antibiotics and external environment compared with the bacteria in planktonic form ${ }^{5}$. Furthermore, ineffective attempts of polymorphonuclear neutrophils penetration into the biofilm may result in periprosthetic osteolysis specifically for orthopaedic implants due to the release of cytotoxic and proteolytic substances ${ }^{6}$.

Therefore, because of such ability of Staphylococcus epidermidis to attach, colonise and form biofilm on the implanted biomaterials surface, it is important to develop and implement therapeutic strategies and effective infection control measures, particularly for the orthopaedic implanted devices $^{2}$. One of the strategies is to coat the orthopaedic implant using Cinnamon oil that has an antimcrobial effect. Cinnamon oil is made from Cinnamonum burminii which is found in Indonesia that also called Indonesian cassia or Cinnamon stick. It is known to have antibacterial effect against several bacteria, such as Streptococcus mutans, Lactobacillus plantarum, and S. Epidermidis ${ }^{7,8,9}$.

Chitosan is a natural bio-degradable and non-toxic biopolysaccharide derived from chittin that is widely used in medicine especially in wound management, drug transporter, and implant coatings. It has biocompatibility, bioadhesive, bacteriostatic, antithrombogenic and osteoconductive properties $^{10,11}$. Nowadays, the bioadhesive properties are promising due to their ability to effectively bind to metal substances, to agglutinate a wide variety of mammalian cells, relative safety to human tissue, and the formulation of film 
Table I: The absorbance level read by ELISA 575nm (A575)

\begin{tabular}{|lcccc|}
\hline & 1st & 2nd & 3rd & Average \\
\hline CIN 2\% & 0.046 & 0.048 & 0.047 & 0.047 \\
CIN 1\% & 0.048 & 0.048 & 0.049 & 0.048 \\
CIN 0.5\% & 0.065 & 0.066 & 0.066 & 0.066 \\
CIN 0.25\% & 0.076 & 0.075 & 0.080 & 0.077 \\
CIN 0.125\% & 0.095 & 0.101 & 0.104 & 0.100 \\
+ve control (S. Epidermidis biofilm only) & 0.054 & 0.057 & 0.059 & 0.057 \\
-ve control (implant without treatment) & 0.036 & 0.039 & 0.039 & 0.038 \\
\hline
\end{tabular}

related to drug delivery systems ${ }^{12,13,14}$. The application of chitosan as bioadhesive can provide a long stay, adequate drug penetration, high efficienct and acceptability ${ }^{15}$. Chitosan also has a wide spectrum antimicrobial activity, however the outcome depends on various factors ${ }^{16,17}$.

Previous study have shown that cinnamon oil coated onto Kwire has an antimicrobial effect in planktonic level of $\mathrm{S}$. Epidermidis? 7 Therefore, this study was expected to be linked and become the advanced research extension of that study to determine the effect of coating stainless steel orthopaedic implants surfaces with cinnamon oil and chitosan as bioadhesive to prevent biofilms formation of S. Epidermidis and to determine the minimum effective concentration of cinnamon oil in chitosan.

\section{MATERIALS AND METHODS}

\section{Bacterial isolates}

Staphylococcus Epidermidis was used as bacterial isolates in this study. It was obtained from body fluid - collected from Sardjito General Hospital,Yogyakarta, Indonesia, and identified in the Microbiology Department, Universitas Gadjah Mada, Yogyakarta, Indonesia. The bacteria were cultured in Trypticase Soy Broth (TSB) media, incubated for 18 to 24 hours at $37^{\circ} \mathrm{C}$, and then suspended into tube filled with $10 \mathrm{cc} \mathrm{NaCL} 0.9 \%$ until similar turbidity with McFarland standard solution was achieved ${ }^{18}$. One hundred microliters of the suspension was taken, added with $9.9 \mathrm{ml}$ TSB media, mixed until homogenous to get bacterial concentration of $10^{6} \mathrm{CFU} / \mathrm{ml}$

\section{Antimicrobials}

Cinnamon oil was made from cinnamon stick (Cinnamomum Burmanii) obtained from local market in Indonesia with authentification by botanical experts. One kilogram of Cinnamon stick and four litres of water were boiled and connected with modified Clavenger device. The device gathered the oil by steam distillation. Sodium Sulphate Exicatus was added to remove watermarks.

This was an in vitro basic science study using rod shaped stainless steel $316 \mathrm{~L}$ orthopaedic implant with $5 \mathrm{~mm}$ diameters and $10 \mathrm{~mm}$ length. It was coarsened using silica sand with grid blasting method to achieve uniformity of coarseness which was confirmed by surface profilometry. The coating was done twice using a mixture of cinnamon oil and chitosan $3 \%$ and $2 \%$ respectively with serial concentration of cinnamon from $0.125 \%$ to $2 \%$. The coated implants were then put into tubes that contained bacterial suspension and incubated in $37^{\circ}$ Celcius within 24 hours. Subsequently, the implants were washed by phosphatebuffered saline (PBS) solution followed by MTT (3-(4,5 dimethylthiazol-2-yl)-2,5-diphenyl tetrazolium bromide) and afterwards isopropanol acid solution was added to dissolve the MTT solution that had reacted with biofilm.

The results were expressed in numbers which represented the absorbance level at ELISA readings on $575 \mathrm{~nm}$ (A575) wavelength. Higher number indicated turbidity resulting from the greater formation of biofilm of the bacteria. The experiment was done three times to seek the average value. For comparison, a tube containing S. Epidermidis biofilm was useed as the standard for biofilm formation, whereas a tube containing TSB only was used as standard for the absence of biofilm formation.

\section{RESULTS}

This study showed that stainless steel implant coated with chitosan and cinnamon oil $2 \%$ and $1 \%$ has lower number, whereas cinnamon oil $0.5 \%, 0.25 \%$, and $0.125 \%$ has higher number compared with the absorbance level of S.Epidermidis biofilm only. It can be concluded that chitosan and cinnamon oil $2 \%$ and $1 \%$ have less biofilm formation of $\mathrm{S}$. Epidermidis compared with tube filled with full $\mathrm{S}$. Epidermidis biofilm.

\section{DISCUSSION}

Coagulase-negative staphylocci constitute the majority of the commensal bacterial microflora and widely distributed throughout the surface of the human body, and therefore it becomes one of the most common species responsible for infection ${ }^{2}$. Biofilm acts as a basic survival mechanism against environmental influences including host immune responses and antimicrobial agents. Moreover, microorganisms within biofilms are less susceptible to antimicrobial treatment compared with their planktonic 
counterparts $^{19}$. Therefore, it is important to prevent formation of biofilm on orthopaedic implanted devices to avoid one of the catastrophic complications in orthopaedic surgery ie. infection $^{6,12,20}$. The use of materials and coatings that release antimicrobials effect can be applied to prevent colonization and consequent biofilm formation. It is considered as one of the practical strategies due to release of antimicrobials effect in lethal concentration for any local planktonic cell before they form biofilm .

Cinnamon is recognised for the presence of cinnalmaldehyde which is highly electronegative and proanthocyanidins that can interfere with biological processes. Both of the components significantly contribute to the antibacterial properties. Due to its safe antibacterial properties, it can be applied as natural food preservatives in many practices ${ }^{21}$. Cinnamon oil has the most potent bactericidal effect against different important pathogens compared to 20 other essential oils used in traditional medicine ${ }^{21,22}$. It has an effective antimicrobial agent including against $\mathrm{S}$. Epidermidis due to biofilm formation inhibition, existing biofilms detachment, and strong bactericidal effect ${ }^{23}$. The effectivity of cinnamon oil in preventing S. Epidermidis biofilm formation has similar effect in terms of its antimicrobial properties compared with antibacterial combination in the form of rifampicin with clindamycin or gentamycin and rifampicin with levofloxacin ${ }^{20}$. In the future, it is expected that cinnamon oil can be used as one of the option in the effort to prevent biofilm formation especially in prosthetic devices.

Previous study about effectiveness of cinnamon oil cream based showed that cinnamon oil has antimicrobial effect against S. Epidermidis biofilm formation when it coated in the K-wire ${ }^{7}$. Compared with that study, this study used chitosan for its adhesive properties. Chitosan can act as good adhesive media due to its abilities to bind with metal. A study conducted by Greene (2008) showed chitosan had the capacity to be sufficiently bonded to stainless steel medical devices. It can also be added with therapeutic antimicrobial agents. The potentiality of chitosan as a coating for orthopaedic devices is proved by biocompatibility, zone of inhibition, and elution tests, whereas functional simulated bone testing suggests the coating strength is sufficient to be used in these applications ${ }^{12}$. Besides that, chitosan can act as a wound dressing material due to its hydrogel-forming properties that have been considered to be advantageous. There are four factors that affect the antimicrobial activity of chitosan. Those factors are microbial factors related to microorganism species and cell age, intrinsic factors of chitosan, physical state, and the environmental factors involving $\mathrm{pH}$ and temperature ${ }^{24}$. It also has an antimicrobial activity againsts Gram-positive and Gram-negative bacteria with lower toxicity towards mammalian cells ${ }^{16,17}$. However, the outcome of its antimicrobial properties is severely affected by various intrinsic and extrinsic factors including the physical states of chitosan. Various physical forms of chitosan and its derivates are used in different areas, whereas those in the forms of beads, films, fibers, and hydrogels are commonly used in biomedical applications ${ }^{24}$.

There are several factors that affect the formation of biofilm onto the surface of biomaterial, including the surface roughness ${ }^{25}$. The biofilm formation and its attachment increases as the increase of high surface free energy and/or wettability due to roughness of the surfaces ${ }^{25,26}$. Stainless steel that was used in this study has high surface free energies and it is usually more hydrophillic. Moreover, rough surfaces tend to show greater bacterial attachment and colonisation due to its hydrophilicity ${ }^{25}$. Therefore, the higher level of absorbance showed in the concentration of cinnamon oil $0.5 \%, 0.25 \%$, and $0.125 \%$ might be due to low to none antimicrobial effect of cinnamon oil and the coating of the stainless steel increase the surface roughness.

Although the findings in this study demonstrate the ability of cinnamon oil and chitosan in preventing biofilm formation of S. Epidermidis, further research regarding the durability of cinnamon oil and chitosan at the implant surface and the effect of cinnamon oil and chitosan in the body tissue should be carried out before it can be applied in the clinical settings.

\section{CONCLUSION}

This study showed that a mixture of cinnamon oil and chitosan as bioadhesive coated on the surface of stainless steel orthopaedic implant has an effect against S.Epidermidis biofilm formation with the minimum cinnamon oil concentration of $1 \%$.

\section{ACKNOWLEDGMENT}

The authors fully acknowedge Titik Nuryastuti MD, $\mathrm{PhD}$ whose help, stimulating suggestions, knowledge, and experience helped us at all times of the study. 


\section{REFERENCES}

1. De Lalla F. Antimcrobial chemotherapy in the control of surgical infectious complications. J Chemother 1999; 11: 440-5.

2. O'Gara JP, Humphreys H, - Staphylococcus Epidermidis biofilms: importance and implications. J Med Microbiol, $2001: 5$ 0; $582-7$.

3. Gustilo RB, Merkow RL, Templeman D. The management of open fractures. J Bone joint Surg Am: 1990; 72: 299-304.

4. Fitzgerald RH. - Total hip arthroplasty sepsis. Prevention and diagnosis. Orthop Clin North Am: 1992; 23: 259-64.

5. Rodriguez-Martinez JM, and A Pascual. Antimicrobial resistance in bacterial biofilms. Rev Med Microbiol. 2006; 17: 65-75.

6. Galanakos SP, Papadakis SA, Kateros K, Papakostas I, Macheros G, -Biofilm and orthopaedic practice: the world of microbes in a world of implants. Orthopaedics and Trauma. 2009; 23: 3.

7. Magetsari R. Effectiveness of Cinnamon oil coating on K-Wire as an antimicrobial agent against Staphylococcus Epidermidis. Malaysian Orthopaedic Journal 2013; 7: 4.

8. Filoche SK, K Soma, and CH Sissons. Antimicrobial effects of essential oil in combination with chlorhexidine digluconate. Oral Microbiol. Immunol. 2005; 20: 221-5.

9. Fabian DM, Sabol K, Domaracka, Bujna'kova D. Essential oils-their antimicrobial activity against Escheria Coli and effect on intestinal cell viability. Toxicol. In Vitro. 2006; 20: 1435-45.

10. Bumgardner JD. Osteointegration of chitosan coated titanium in rabbit tibia. Implant surface coatings and new materials. 2007; 10: 22 .

11. Hafdani FN, Sadeghinia N, A review on application of chitosan as a natural antimicrobial. World Academy od Science, Enginering and Technology. 2001; 5; 2-23.

12. Greene AH, Bumgardner JD, Yang Y, Moseley J, Haggard WO- Chitosan-coated Stainless Steel Screws for Fixation in Contaminated Fractures. Clin Orthop Relat Res. 2008; 466: 1699-704.

13. Peh K, Khan T, Ching H, Mechanical, bioadhesive strength and biological evaluations of chitosan films for wound dressing. $J$ Pharm Pharm Sci 2000; 3(3): 303-11.

14. Suknuntha K., Tantishaiyakul V, Worakul N, Taweepreda W, Characterization of muco- and bioadhesive properties of chitosan, PVP, and chitosan/PVP blends and release of amoxicillin from alginate beads coated with chitosan/PVP. Drug Dev Ind Pharm. 2011; 37(4): 408-18.

15. Varshosaz J, Jaffari F, Karimzadah S, Development of biodhesive chitosan gels for topical delivery of lidocaine. Scientica Pharmaceutica Scip. Pharm. 2006; 74: 209-23.

16. Franklin TJ, Snow GA, Biochemistry of Antimicrobial Action, 3rd ed. Chapman and Hall, London, 1981; p.175.

17. Takemono, K., Sunamoto, J., Askasi, M., Polymers and Medical Care. Mita, Tokyo;1989; Chapter IV

18. Murray PR, Baron EJ, Pfaller MA, Tenover FC, Yolken RH, - Manual of Clinical Microbiology sixth edition. ASM Press: 1995; Washington DC.

19. Brown MRW, P Gilbert. Sensitivity of biofilms to antimicrobial agents. J. Appl. Bacteriol. 1993; 74: 87-97.

20. Gomes F, Teixeira P, Ceri H. Oliveira R - Evaluation of antimicrobial activity of certain combinations of antibiotics against in vitro Staphylococcus epidermidis biofilms. Indian J Med Res 2012; 135: 542-7.

21. Shan B, Cai John YZ, Corke H. - Antibacterial properties and major bioactive components of cinnamon stick (Cinnamon burmannii): activity against foodborne pathogenic bacteria. Journal of Agricultural and Food Chemistry. 2007; 55: 5484-90.

22. Prabuseenivasan S, Jayakumar M, Ignacimuthu S. In vitroantibacterial activity of some plant essential oils. BMC Complementary and Alternative Medicine. 2006; 6: 39.

23. Nuryastuti T, van der Mei HC, Busscher HJ, et al Effect of cinnamon oil on icaAexpression and biofilm formation by Staphylococcus epidermidis. Applied and Environmental Microbiology. 2009; 75: 6850-5.

24. Kong M, Chen XG, Xing K, Park HJ, Antimicrobial properties of chitosan and mode of action: A state of the art review. International Journal of Food Microbiology. 2010; 144: 51-63.

25. Kokare CR, Chakraborty S, Khopade AN, Mahadik KR, Biofilm: Importance and applications. Indian Journal of Biotechnology 2009: 8; 159-68.

26. Donlan RM, Biofilms: Microbial life on surfaces, Emerg Infect Dis 2002; 8: 881-90. 